\title{
Experimental Tuning of the AIFSN Parameter to Prioritize Voice Over Data Transmission in 802.11E WLAN Networks
}

Miroslaw Narbutt

Technological University Dublin, miroslaw.narbutt@tudublin.ie

Mark Davis

Technological University Dublin, mark.davis@tudublin.ie

Follow this and additional works at: https://arrow.tudublin.ie/commcon

Part of the Systems and Communications Commons

\section{Recommended Citation}

Narbutt, M. \& Davis, M. (2007) Experimental tuning of the AIFSN parameter to prioritize voice over data transmission in 802.11E WLAN networks. ACM International Conference on Wireless Communciations and Mobile Computing (IWCMC 2007), Hawaii, August, 2007.

This Conference Paper is brought to you for free and open access by the Communications Network Research Institute at ARROW@TU Dublin. It has been accepted for inclusion in Conference papers by an authorized administrator of ARROW@TU Dublin. For more information, please contact arrow.admin@tudublin.ie, aisling.coyne@tudublin.ie,gerard.connolly@tudublin.ie. 


\title{
EXPERIMENTAL TUNING OF THE AIFSN PARAMETER TO PRIORITIZE VOICE OVER DATA TRANSMISSION IN 802.11E WLAN NETWORKS
}

\author{
Miroslaw Narbutt and Mark Davis \\ narbutt@cnri.dit.ie,mark.davis@dit.ie \\ Communications Network Research Institute, \\ School of Electronic and Communications Engineering, \\ Dublin Institute of Technology, Ireland
}

\begin{abstract}
In this paper we experimentally study the impact of one EDCA parameter, namely AIFSN on a mixed voice/data wireless transmission. In particular we investigate how the tuning of this parameter affects both the voice transmission quality and background data throughput. We predict end-to-end voice transmission quality from time varying transmission impairments using the latest Appendix to the ITU-T E-model. Our experimental results show that the tuning of the AIFSN parameter can successfully prioritize voice transmission over data in a real 802.11e network. To the best of our knowledge, this is the first experimental investigation on tuning of MAC layer parameters in a real 802.11e WLAN network and its effect on end-to-end voice transmission quality.
\end{abstract}

Index Terms - speech communication, data transmission, wireless LAN, voice transmission quality, IEEE802.11e WLAN.

\section{INTRODUCTION}

Real-time voice transmission over wireless LAN (VoWLAN) imposes stringent requirements on transmission impairments such as end-to-end delays, jitter, and packet loss. The responsibility of meeting these requirements is shared between the various communication layers. Actions at the application layer include efficient encoding and packetization schemes, packet loss concealment (PLC) techniques, adaptive dejitter buffering, echo cancellation, etc. On the network side, the new IEEE 802.11e protocol supports voice traffic by differentiating channel access probability among different traffic categories. In particular, the new, extended channel access mechanism (EDCA) allows for adjusting a number of channel access parameters at the L2/MAC layer to prioritize VoIP packets over other traffic types.

Application-layer adaptation mechanisms and MAC-layer parameters tuning can greatly mitigate the effect of transmission impairments and thus improve speech transmission quality. However, these mechanisms are often complex and difficult to tune properly. We claim that if a part of the VoIP transmission path is being tuned, the impact of local tuning actions on the whole end-toend (mouth-to-ear) transmission has to be taken into account. For this reason we developed a method for evaluating user satisfaction regarding end-to-end VoIP transmission quality from time varying transmission impairments. This method has shown to be particularly effective in evaluating various playout buffer algorithms [1, 2], assessing VoIP performance in Voice over WLAN systems $[3,4,5]$, and was recently standardized by the ITU-T [6] as an Appendix to [7].

In this paper we use this method to experimentally evaluate the capability of the EDCA mechanism to support voice traffic in a mixed voice/data transmission over 802.11e WLAN. We investigate how real-time voice can be supported by tuning one EDCA parameter, namely AIFSN and how this impacts background data transmission. This paper is structured as follows. In Section 2, the 802.11e WLAN experimental setup is described, EDCA mechanism is outlined and proper dejitter buffering at application layer is addressed. Experimental results are presented and discussed in Section 3. Finally, the paper is concluded in Section 4.

\section{802.11E WLAN EXPERIMENT}

\subsection{Experimental testbed.}

The 802.11e wireless/wired test bed consists of 15 desktop PCs acting as wireless VoIP terminals, one desktop PC acting as a background traffic generator, and one desktop PC acting as an access point (AP). All machines in the test bed use 802.11 PCMCIA wireless cards based on Atheros chipsets controlled by MadWiFi wireless drivers and Linux OS (kernel 2.6.9). The MadWiFi drivers (Release 0.9.1 and above) provide working implementation of IEEE 802.11e EDCA mechanism [8]. All of the nodes are also equipped with a $100 \mathrm{Mbps}$ Ethernet cards. The PC that acts as access point routes traffic between the wired network and the wireless clients, and vice versa (each PC has two interfaces: one on the wireless and one on wired network). During the experiments each VoIP terminal runs one VoIP session and all sessions are bi-directional. In this way each terminal acts as the source of an uplink flow (i.e. sender) and the sink (i.e. receiver) of a downlink for a VoIP session. The wired interface off one PC is used to generate background traffic which is routed via the AP to 
the wireless interface of the same PC. All generated traffic involved a wired and a wireless interface so that no traffic was generated between wireless interfaces. The wireless stations were located within 5 meters range from the AP to ensure that the wireless link quality is good. This test bed is illustrated in Fig. 1. Voice traffic was generated using RTPtools [9] which generated G.711 encoded voice packets (80bytes audio frames created every $10 \mathrm{~ms}$ ) with fixed IP packet overhead of 12 bytes for RTP, 8bytes for UDP, and 20 bytes for IP layer.

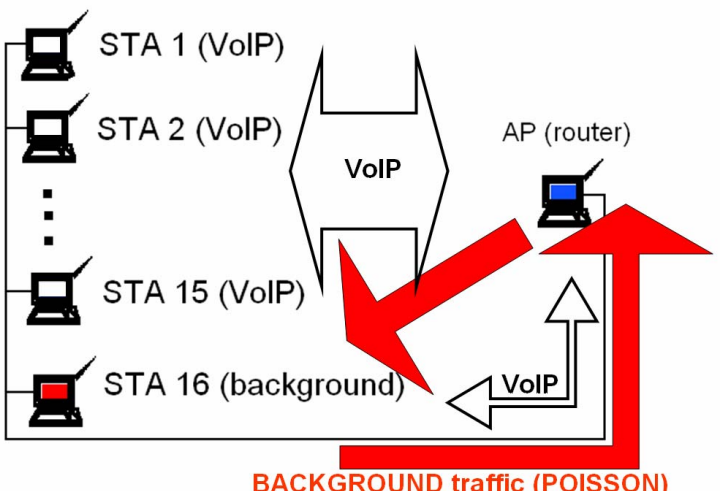

Figure 1. Experimental 802.11e test bed

During the experiments bi-directional transmission of packets was realized in the form of alternating active and passive periods modeled as a four state Markov chain (talker A active, talker B active, both active, both silent). The duration of states and the transitions between them followed the ITU-T recommendation P.59. [10]. This resulted in an ON-OFF modulated CBR traffic stream being generated. Background traffic in the form of Poisson distributed UDP packet flow was generated using MGEN traffic generator [11]. For the experiments we used 1, 2, and 4Mbps background traffic. To measure effective throughput (goodput) of the background traffic we used TRPR package [12]. The size and sending rate of the IP packets comprising the load is specified in Table I.

Table 1. The size and sending rate of the packets comprising the background load

\begin{tabular}{cccc}
\hline $\begin{array}{c}\text { IP packet } \\
\text { size [Bytes] }\end{array}$ & $\begin{array}{c}\text { 1Mbps load } \\
\text { [pps] }\end{array}$ & $\begin{array}{c}\text { 2Mbps load } \\
\text { [pps] }\end{array}$ & $\begin{array}{c}\text { 4Mbps load } \\
\text { [pps] }\end{array}$ \\
\hline 256 & 488 & 977 & 1954 \\
512 & 244 & 488 & 977 \\
1024 & 122 & 244 & 488 \\
1500 & 83 & 167 & 336 \\
\hline
\end{tabular}

The reasoning behind choosing UDP and not TCP as a transport protocol for carrying background traffic is threefold: 1) UDP background traffic gives more accurate estimate of the actual load in the network (no retransmissions at transport layer) 2) results obtained with UDP constitute an upper bound for the throughput possible with TCP; 3 ) retransmissions of lost or corrupted packets is done by the 802.11 MAC-layer so TCP do net get affected by the packet loss [13].
During experiments all the measured VoIP data (packet arrival times, timestamps, sequence numbers, and marker bits) was collected at all receiving terminals to be processed later (off-line) by a program that simulated the behavior of the de-jittering buffer. Finally, the quality assessment algorithm was used to predict the quality of voice transmission.

\subsection{MAC-layer parameters tuning}

The newer IEEE standard 802.11e offers two modes of MAC operation: contention-based channel access called Enhanced Distribution Coordinate Access (EDCA) and contention-free channel access called Hybrid Controlled Channel Access (HCCA). In our experiments we have focused on the performance of the EDCA mode that differentiates the channel access probability among different traffic categories (TCs). When this operational mode is used, the packets are categorized in different TCs, and later mapped to four prioritized output queues called access categories (ACs). Each AC uses a set of parameters that controls the access probability to the wireless medium:

- $\quad$ IIFSN parameter controls the idle time (i.e. the the arbitration interframe space, AIFS) after which a transmission may occur;

- $\quad C W \min$ and $C W \max$ parameters define the range of the contention window $(\mathrm{CW})$ values from which the back-off time is randomly selected;

- TXOP parameter controls the time interval for which a station holds the channel (transmission opportunity) allowing for multiple packet transmission on a single channel access opportunity.

Configuring these parameters for each AC separately enables access probability differentiation between TCs. Since a station with packet to send must wait until the medium is idle and then wait for an additional period of time AIFS, the AIFSN parameter for the voice AC VO $\left(A I F S N_{\left[A C_{-} V O\right]}\right)$ should be smaller than the $A \overline{I F S N}$ parameter for the background AC BK $\left(A I F S N_{[A C B K]}\right)$. In this way time-sensitive voice traffic will contend sooner for accessing the wireless medium and thus will on average more transmission opportunities over the lesssensitive background traffic. After the AIFS period, the stations with a packet to send select random numbers between the CWmin and CWmax for each contending AC. Since the smallest number indicates "the winner", the values of $C W \min$ and $C W \max$ should be lower for the voice queue than for the background queue. In general the combination of AIFSN, CWmin and CWmax should be configured so that high-priority voice packets win transmission opportunities over background traffic. However, to avoid situations in which the low-priority traffic is completely blocked, the sum of AIFSN plus CWmax for high-priority voice should be greater than AIFSN for low-priority traffic. In our experiments the voice packets were mapped into the voice AC (AC_VO) queue while the data traffic was mapped into the 
background (AC_BK) queue based on their TOS values in IP packets' headers.

During the experiment we prioritized voice over background traffic by increasing the number of time slots comprising the background AIFS period $\left(A I F S N_{\left[A C_{-} B K\right]}\right)$ from 2 to 15 slots. All the other AC BK parameters were: $C W \min =7, C W \max =1023, T X O P=0$ and they were kept fixed for the duration of the first experiment. The parameters under consideration for both $\mathrm{AC}$ BK and AC_BK are listed in Table II

Table 2. EDCA parameters settings during the experiments

\begin{tabular}{ccc}
\hline $\begin{array}{c}\text { Access } \\
\text { Parameter }\end{array}$ & $\begin{array}{c}\text { AC_VO } \\
\text { (STAs and AP) }\end{array}$ & $\begin{array}{c}\text { AC_BK } \\
\text { (STAs and AP) }\end{array}$ \\
\hline CWmin & 7 & 7 \\
CWmax & 1023 & 1023 \\
$A I F S N$ & 2 & $2,3, \ldots 14,15$ \\
TXOP & 0 & 0 \\
\hline
\end{tabular}

\subsection{Application-layer parameters tuning}

In our experiments we used Ramjee's algorithm [14] which is often used as a reference playout buffer controller. The algorithm uses the same playout delay throughout a given talkspurt but permits different playout delays for different talkspurts. We modified the original Ramjee's algorithm by adding one parameter, namely playout_offset that represents additional pre-buffering delay. In our solution the playout time $p_{i}$ at which the the $i$-th packet, assumed to be the first packet in a talkspurt (played at the destination) is calculated as follow:

$$
p_{i}=t_{i}+\hat{d}_{i}+\beta \cdot \hat{v}_{i}+\text { playout_offset }
$$

where $\hat{d}_{i}$ and $\hat{v}_{i}$ are the estimates of delay of the $i$-th packet delay $n_{i}$ and its variance respectively and are calculated as follows:

$$
\begin{gathered}
\hat{d}_{i}=\alpha \cdot \hat{d}_{i-1}+(1-\alpha) \cdot n_{i} \\
\hat{v}_{i}=\alpha \cdot \hat{v}_{i-1}+(1-\alpha) \cdot\left|\hat{d}_{i}-n_{i}\right|
\end{gathered}
$$

Parameter $\beta$ controls the delay/packet loss ratio while parameter $\alpha$ controls the ability of the algorithm to follow the changes in the delay. By experimenting with different values of $\alpha, \beta$, and playout_offset in a real wireless environment we were able to chose the values (i.e. $\alpha=$ $0.998002, \beta=2$, playout_offset $=40 \mathrm{~ms}$ ) that maximized rating factor $R$ for all possible AIFSN and CWmin settings.

\section{EXPERIMENTAL RESULTS}

Experiments covered 3 background traffic loads (1, 2, 3Mbps) 4 packetization schemes for background (256Bytes, 512Bytes, 1024Bytes and 1500Bytes packets)
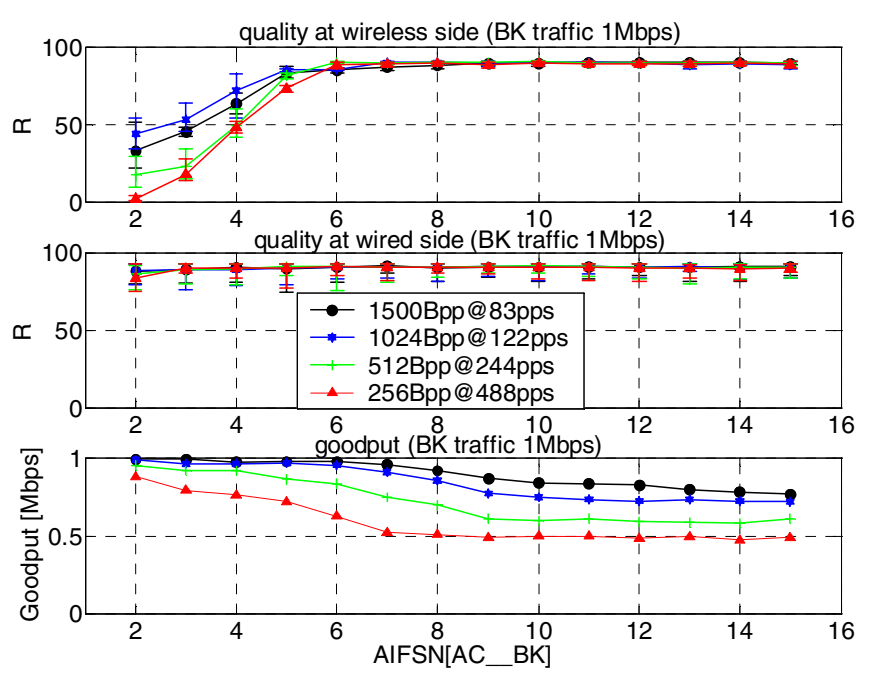

Figure 2. Quality of voice transmission and effective throughput of $1 \mathrm{Mbps}$ background traffic vs IIFSN $_{\left[A C_{-} B K\right]}$
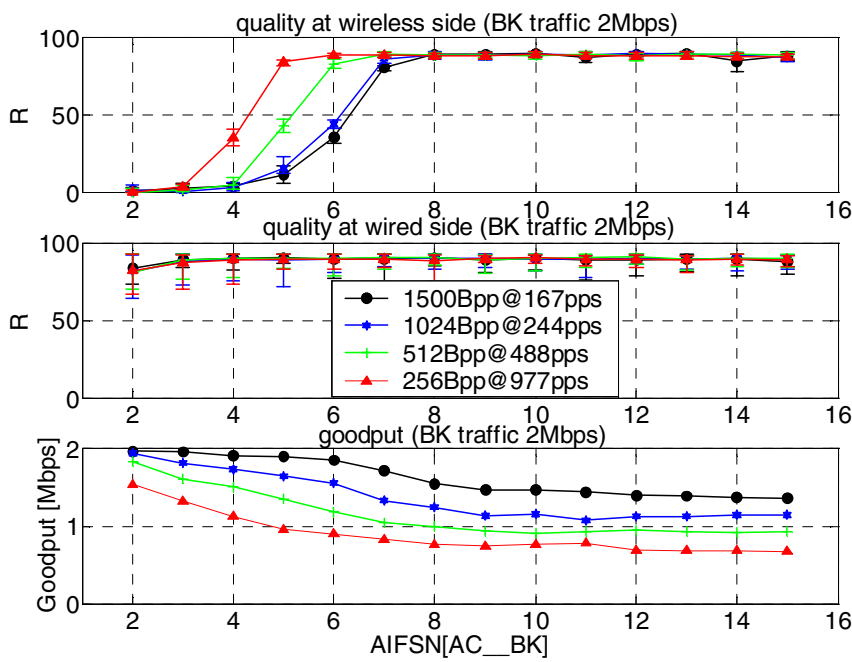

Figure 3. Quality of voice transmission and effective throughput of $2 \mathrm{Mbps}$ background traffic vs $\operatorname{AIFSN}_{\left[A C_{-} B K\right]}$
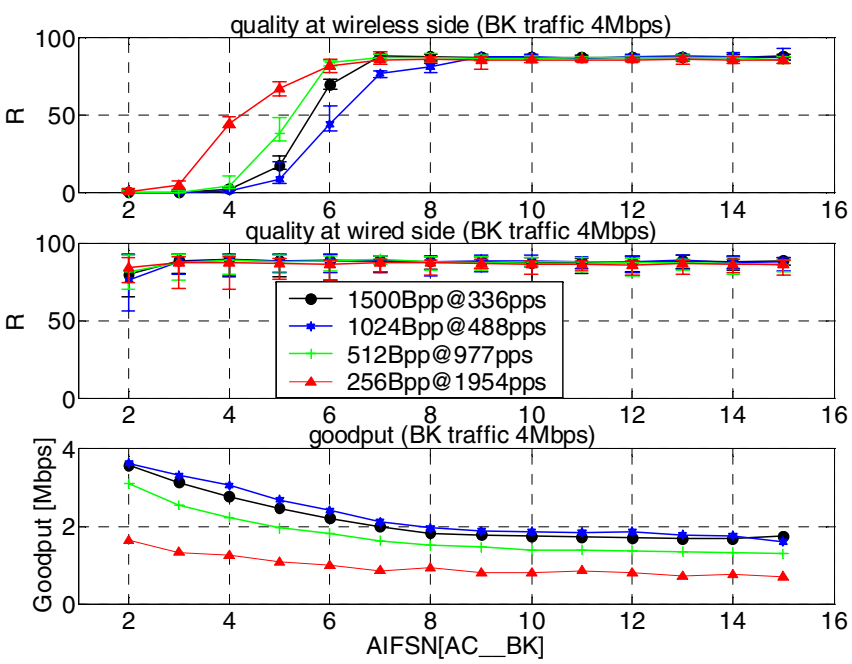

Figure 4. Quality of voice transmission and effective throughput of $4 \mathrm{Mbps}$ background traffic vs $\operatorname{AIFSN}_{\left[A C_{B} B K\right]}$ 
and 14 settings of the $A I F S N_{\left[A C_{-} B K\right]}$ parameter: $2,3 \ldots 14$, and 15 time slots. Figures 2, 3 and 4 shows the average voice transmission quality (at wireless and wired interface) calculated for all 15 VoIP terminals and effective throughput (goodput) as a function of $A I F S N_{\left[A C_{-} B K\right]}$ for three background traffic loads (1Mbps, $2 \mathrm{Mbps}$, and $4 \mathrm{Mbps}$ respectively).

It can be seen that voice transmission at thte wireless network can be effectively prioritized over data by tuning the $A I F S N_{[A C B K]}$. Increasing $\operatorname{AIFSN}_{[A C B K]}$ essentially promotes the $\mathrm{AC}$ VO queue at the expense of the $\mathrm{AC} \_\mathrm{BK}$ queue in terms of probability access.

The bigger the difference in $A I F S N$ values, the easier it is for the $\mathrm{AC}_{-} \mathrm{VO}$ queue to win transmission opportunities from the $\overline{A C} B K$. As a result, transmission impairments (delay, jitter and packet loss) are reduced and the overall transmission quality is improved. For example, when the AIFSN difference between AC_VO and AC_BK was 6 $\left(\right.$ AIFSN $_{\left[A C_{-} B K\right]}=8$ and $\left.A I F S N_{\left[A C_{-} V O\right]}=2\right)$, all VoIP stations could experience at least "tool" voice transmission quality (indicated by $R \geq 70$ ) for all examined background traffic loads and packetization schemes. Conversely a substantial reduction in the background traffic goodput was observed. In some cases (i.e. the 256 Bytes background packets load) the goodput of the background traffic was almost halved. Increasing the AIFSN difference between $\mathrm{AC} \_\mathrm{BK}$ and $\mathrm{AC}$ _VO further penalizes background traffic by making it more difficult to win transmission opportunities.

\section{CONCLUSIONS}

In this paper we have experimentally evaluated the capability of the new 802.11e MAC protocol to support voice calls in a mixed voice/data transmission over WLANs. In our experiments we have focused on the contention-based mode of MAC operation called Enhanced Distributed Channel Access (EDCA) and more specifically on one quality enhancement parameter that controls the arbitration interframe space (AIFS), i.e. AIFSN parameter.

Our results show that the tuning of the AIFSN parameter can improve the quality of voice transmission at the wireless network while reducing the goodput of the background data traffic. For example, when the AIFSN difference between $\mathrm{AC} \_\mathrm{VO}$ and $\mathrm{AC} \_\mathrm{VO}$ was 6 $A I F S N_{\left[A C_{-} B K\right]}=8$ slots and $\left.\operatorname{AIFSN}_{\left[A C_{-} V O\right]}=\overline{2}\right)$, all fifteen VoIP terminals could experience at least "toll" voice transmission quality (indicated by $\mathrm{R} \geq 70$ ) in the presence of the heavy background traffic injected to the network.

The AIFSN differentiation is an effective mechanism for voice prioritization over data because of the very existence of discrete instants of times (protected slots represented by the AIFSN difference) where a lower number of stations may compete and access the channel. This increases the effectiveness of the overall random access mechanism for the high-priority stations [15].
To our knowledge, this is the first experimental demonstration of voice prioritization in the real 802.11e WLAN network from the perspective of end-to-end speech transmission quality and end user satisfaction.

\section{ACKNOWLEDGMENTS}

This work was supported by Science Foundation Ireland grant 03/IN3/I396.

\section{REFERENCES}

[1] Miroslaw Narbutt, Andrew Kelly, Liam Murphy, Philip Perry, "Adaptive VoIP Playout Scheduling: Assessing User Satisfaction," IEEE Internet Computing Magazine, vol. 09, no. 4, July/August ' 05 .

[2] Miroslaw Narbutt, Mark Davis, "Assessing the Quality of VoIP Transmission Affected by Playout Buffer Scheme," Proc. of the ETSI/IEE Measurement of Speach and Audio Quality in Networks Conference 2005 (MESAQIN 2005), Prague, June ' 05.

[3] Miroslaw Narbutt, Mark Davis, "An Assessment of the Audio Codec Performance in Voice over WLAN (VoWLAN) Systems," Proc. of the International Conference on Mobile and Ubiquitous Systems: Networking and Services, (MOBIQUITOUS 2005), San Diego, July ' 05.

[4] Miroslaw Narbutt, Mark Davis "Gauging VoIP Call Quality from 802.11b Resource Usage", Proc of the IEEE International Symposium on a World of Wireless, Mobile and Multimedia Networks (WoWMoM06), Buffalo-NY, June '06 [5] Miroslaw Narbutt, Mark Davis, "Experimental investigation on VoIP performance and the resource utilization in 802.11b WLANs", Proc of the 31st IEEE Conference on Local Computer Networks (LCN'06), Tampa, November '06

[6] ITU-T Recommendation G.109 Appendix I (01/2007) "The E-model based quality contours for predicting speech transmission quality and user satisfaction from time-varying transmission impairments"

[7] ITU-T Rec. G.109 "Definition of categories of speech transmission quality", September '99

[8] H. Yoon, "Test of MADWIFI-ng WMM/WME inWLANs", TR nr 1, February '06

[9] RTPtools:

http://www.cs.columbia.edu/IRT/software/rtptools

[10] ITU-T Recommendation P.59, "Artificial conversational speech", March '93

[11] MGEN, The Multi-Generator Toolset: http://pf.itd.nrl.navy.mil/mgen/

[12] TRace Plot Real-time package (TRPR) http://pf.itd.nrl.navy.mil/protools/trpr.htm

[13] S. Garg, M. Kappes "An Experimental Study o f Throughput for UDP and VoIP Traffic in IEEE 802.11b Networks" , Proc of the IEEE Wireless Communications and Networking Conference, WCNC 2003, New Orelan, '03

[14] R. Ramjee, J. Kurose, D. Towsley, and H. Schulzrinne, "Adaptive playout mechanisms for packetized audio applications in wide-area networks", Proc. of the IEEE INFOCOM, Toronto, ' 99

[15] G. Bianchi, I. Tinnirello, L. Scalia, "Understanding $802.11 \mathrm{e}$ contention-based prioritization mechanisms and their coexistence with legacy 802.11 stations" IEEE Network 19(4): 28-34 (2005) 